This item was submitted to Loughborough's Research Repository by the author.

Items in Figshare are protected by copyright, with all rights reserved, unless otherwise indicated.

\title{
Modelling spectral irradiation effects on single- and multijunction amorphous silicon photovoltaic devices
}

PLEASE CITE THE PUBLISHED VERSION

http://dx.doi.org/10.1109/PVSC.2002.1190833

PUBLISHER

(C) IEEE

VERSION

VoR (Version of Record)

LICENCE

CC BY-NC-ND 4.0

\section{REPOSITORY RECORD}

Betts, Thomas R., Ralph Gottschalg, and David Infield. 2019. "Modelling Spectral Irradiation Effects on Singleand Multijunction Amorphous Silicon Photovoltaic Devices”. figshare. https://hdl.handle.net/2134/8215. 
This item was submitted to Loughborough's Institutional Repository (https://dspace.lboro.ac.uk/) by the author and is made available under the following Creative Commons Licence conditions.

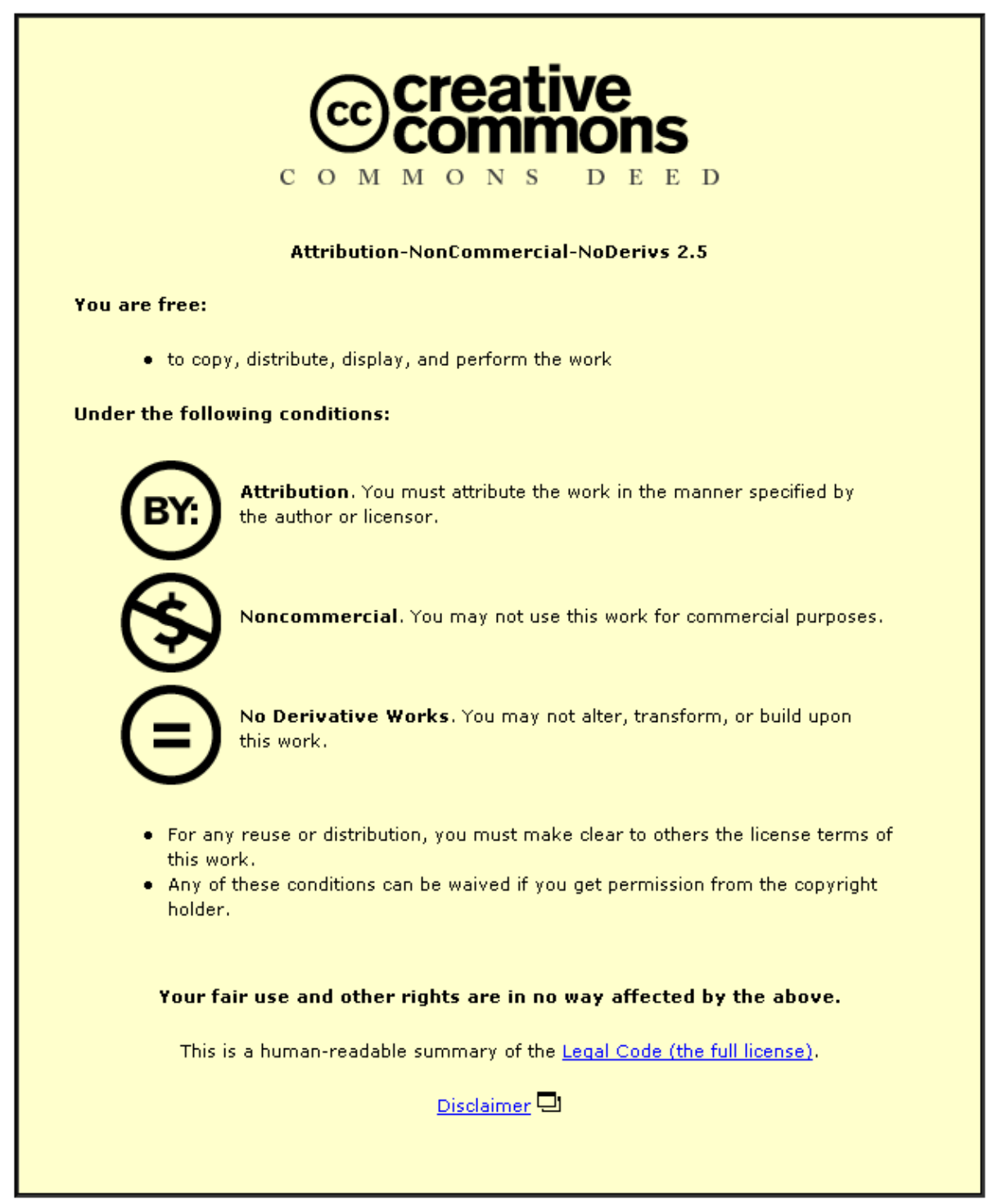

For the full text of this licence, please go to: http://creativecommons.org/licenses/by-nc-nd/2.5/ 


\title{
MODELLING SPECTRAL IRRADIATION EFFECTS ON SINGLE- AND MULTI- JUNCTION AMORPHOUS SILICON PHOTOVOLTAIC DEVICES
}

\author{
T. R. Betts, R. Gottschalg, D. G. Infield \\ Centre for Renewable Energy Systems Technology, Loughborough University, Loughborough, LE11 3TU, UK
}

\begin{abstract}
It has been previously reported that variations in the spectral irradiance under which an amorphous silicon device operates can have a significant effect on its electrical performance, often contributing to enhanced system yields compared to crystalline-based systems.

In this work, spectral irradiance data based on models and measurements taken at the Centre for Renewable Energy Systems Technology (CREST) in the UK are presented. These are input into electrical models for amorphous silicon devices incorporating different number of junctions in order to investigate the impact of changing spectral irradiation. The results can be classified broadly as primary effects, those accounting for the available spectrally useful irradiance and secondary effects that consider the effects of mismatched currents in the stacked cells of multi-junction devices. The modeled short circuit currents correlate well with measurements and are demonstrated as a useful tool for further investigation.
\end{abstract}

\section{INTRODUCTION}

The environmental conditions in which a photovoltaic device operates vary greatly with time, season and location and always differ, most often substantially, from the standard laboratory test conditions (cell temperature $25^{\circ} \mathrm{C}$, irradiance $1000 \mathrm{~W} / \mathrm{m}^{2}$, spectrum AM1.5G). This has a large impact on the performance of installed systems, leading to measured outputs that are impossible to anticipate based only on the nameplate efficiency and measurements of global irradiation.

Changes in efficiency due to temperature, total irradiance and incident light spectrum have been observed and quantified for various technologies [1-3]. Effects due to temperature are now included in many commercial system modeling packages, yet those due to irradiance (both total and spectral) are mostly ignored. This can be attributed to the dominance of crystaltine silicon (c-Si) in the photovoltaic market. Compared to amorphous silicon (aSi) the low fight response is relatively weak, so times when these conditions prevail are ignored as being energetically irrelevant. Also, the broader spectral response of c-Si (typically 300-1100 $\mathrm{nm}$ due to the reduced band-gap) makes these devices less sensitive to variation in the spectrum, instead temperature is the dominant environ- mental factor affecting performance. This is not so for a$\mathrm{Si}$, which has a much smaller temperature coefficient and a larger band-gap, leading to greater influence by the spectrum. Indeed we believe that seasonal performance variation in a-Si devices previously attributed to seasonal degradation/thermal annealing cycles is for the most part due to spectral effects. This variation in output is important to understand since it has implications for sizing stand-alone systems and calculating monthly revenue streams for grid-connected installations.

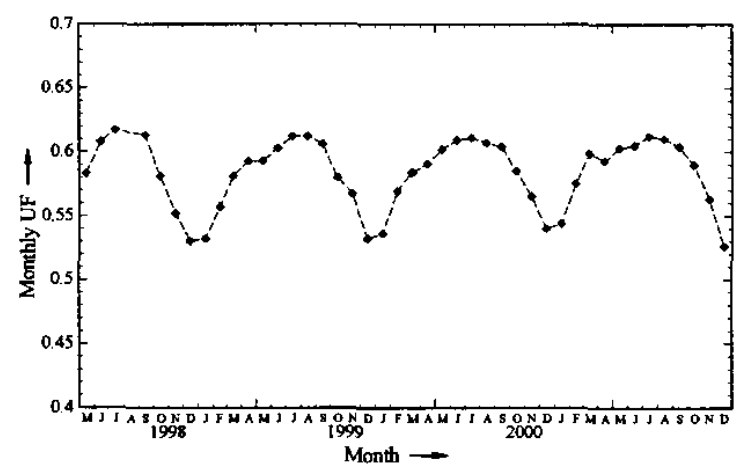

Fig. 1. Seasonal variation of the UF at Loughborough.

Spectral effects become even more important when considering multi-junction devices. Recent studies have shown a-Si devices built from stacked sub-cells to exhibit system yields and performance ratios similar to and even exceeding those of $\mathrm{c}$-Si devices with much higher nameplate efficiencies, [4]. This can be attributed to all three of the environmental effects mentioned - the favorable temperature response, the low light response, which can be significant in cloudy locations, and seasonal spectral effects. It has been observed [5-7] that these spectral effects contribute significantly to the enhanced yields in summer due to better spectral matching, which coincides with the increased resource in these months. The magnitude of spectral effects depends strongly on location, which reflects on both airmass variations and weather. The effects become more noticeable further from the equator where the seasonal change in airmass is more pronounced and cloud formations tend to be denser and more frequent. Spectral effects can be very important for 
certain locations, as shown in Fig. 1. It is helpful for estimating the significance of spectral effects to define a useful fraction (UF) of the incident irradiance as the proportion of irradiation within the spectrally responsive region of the device under investigation relative to the total irradiance (which is due to our instrumentation up to a maximum wavelength of $1700 \mathrm{~nm}$ ). It is apparent from, that the monthly useful fraction varies significantly throughout the year, corresponding to a change of about $15 \%$ around the annual average value of UF. The pattern is quite consistent over the measurement period of 44 months. These changes will have a direct influence on the operating efficiency of solar cells. Furthermore, the constantly changing spectrum will lead to persistently mismatched cells in a double junction device.

This work presents the results of modeling these spectral effects and their impact on the electrical performance of amorphous silicon devices as installed at Loughborough, UK. It is shown through measurement and then simulation of solar spectra, that there are significant deviations from the AM1.5 standard spectrum which is normally used in calculations.

\section{APPROACH}

These spectra are used as input into a photocurrent model to show the influence of spectral variation on the electrical output of a device. This approach is applied to various single and multi-junction a-Si devices produced by RWE Solar, Division Phototronics, with adjustments being made to the electrical model accordingly. The results of the model are then validated against module and spectrat measurements taken at Loughborough.

\section{Spectral Model}

The factors affecting attenuation of solar radiation in the atmosphere fall into two categories: geometric path length through the atmosphere, and constitution of the atmosphere. The first depends on the position of the Sun relative to the point of interest on Earth and can be calculated with the latitude, longitude, time of day, and time of year as inputs. The second depends on the local climate type (urban, rural, maritime, mountain, etc.) and the weather (predominantly the moisture content of the air and the amount and type of cloud). These factors combine to give the overall wavelength-dependent transmission of solar radiation through the atmosphere and as each of the factors changes, so does the spectral content of light reaching the ground.

The spectral modeling is performed in two sections. First, a clear-sky spectrum is generated, with beam and diffuse components modeled separately. This includes attenuation in the bulk of the atmosphere due to scattering and absorption processes by particles and gases, but assumes a cloud-free sky. There are a small number of clear-sky spectral models currently in use, some rigorous codes involving many inputs and knowledge of the structure of the atmosphere, and another class of simple models, based on extinction processes modeled with Bouger's Law, equation (1).

$$
I=I_{0} e^{-\infty x}
$$

where $l$ is the intensity of radiation, $l_{0}$ is the intensity before passing through the absorbing medium (the atmosphere), $\alpha$ is the extinction coefficient, and $x$ is the thickness traversed.

The model used here belongs to the latter category, since these are far more transferable from one site to another and involve inputs which are more widely available or else uncomplicated to measure. Each of these models works on a similar basis and the general principles should be applicable to any climate once suitable changes have been made to the sub-models (eg: for aerosols). On these grounds, the clear-sky model presented here is an extension to that of Bird and Riordan [8], incorporating updated datasets, absorption models, and optical mass calculations, and changes to the aerosol model based on published UK measurements.

Fig. 2 shows the overall structure of the spectral model, including the components used for the initial fitting process.

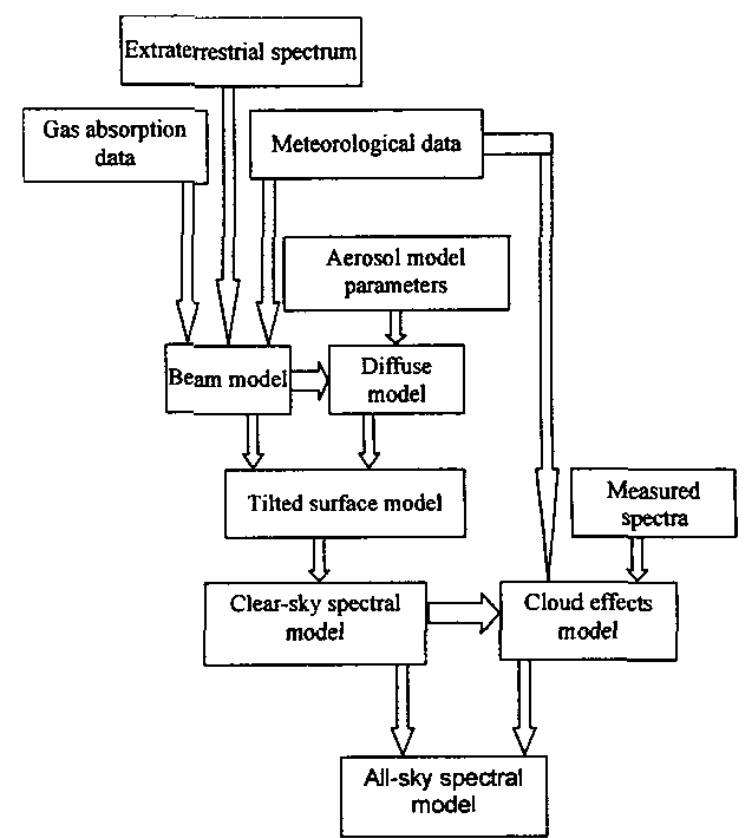

Fig. 2. Spectral model components.

To complete the spectral model, the effects of cloud are introduced. This is a complex influence to model since the amount of cloud present can vary rapidly (c.f. air temperature which changes in a much slower, more predictable way). The only simple, widely available parameter with which to characterize the cloud amount is the total irradiance (or the clearness index, $\mathrm{k}_{\mathrm{T}}$, the ratio of ground-level measured irradiance to irradiance at the top of the atmosphere). Within the limitations of a simple model, designed to use only readily available meteorological data inputs, it is not possible to model cloud effects from a purely physical basis. Therefore, this influence is accounted for by 
modifying the clear-sky spectrum by a function of clearness index (and airmass, since $k_{T}$ has an AM dependence) whose parameters are fitted in a one-off process using measured spectral data.

Finally, the inputs required by the spectral model are: ambient air temperature, air pressure, relative humidity, and global, total (broadband) irradiance in the plane of the collector array. When this data is unavailable, or for projection purposes, a commercial data simulation package (such as Meteonorm) can be used to provide a reasonable estimates at monthly timescaies.

\section{Device Model}

The key electrical device parameter to be modeled is the short circuit current $\left(l_{\mathrm{sc}}\right)$, as this is the characteristic most affected by spectral effects, [9]. Concentrating on this parameter allows the analysis to side-step the voltage dependence of the photocurrent $\left(I_{\mathrm{ph}}\right)$ as well as device quality issues. Minor effects due to the resistance terms are neglected in what follows. The photocurrent of a single junction can be modeled, given knowledge of the spectral response of the device. This straightforward multiplication accounts for what we term the primary spectral effect, an effect that depends on the availability of the spectrally useful irradiance. It does, however, not account for effects which are due to stacked cells in a multijunction device. The latter effect is termed secondary spectral effect; it is superimposed on the primary spectral effect and also depends on the spectral composition of the light.

The photocurrent of a single cell is modeled by combining the known spectral response of the device with the irradiance spectrum to which it is subjected and integrating over all wavelengths where the device has a non-zero response, as given in equation (2).

$$
I_{s c}=I_{p h}=A \times \int_{h_{1}}^{\lambda_{2}} S R(\lambda) G(\lambda) d \lambda
$$

where $G(\lambda)$ is the spectral irradiance incident in the plane of the device $\left(W / m^{2} n m\right), S R(\lambda)$ is the spectral response of the device referenced to a single cell (AM), and $A$ is the cell area $\left(\mathrm{m}^{2}\right)$. The cell is responsive to light in the band from $\lambda_{1}$ to $\lambda_{2}$. To simulate the output of the actual samples under test at CREST, the cell current generated with equation (2) is multiplied by the relevant number of parallel strings for the tested mini-modules in question.

For application to multi-junction devices, the above approach is not wholly satisfactory, as there are two cells with different quantum efficiencies connected in series. One needs to account for mismatched series connected cells, as a shift in the spectrum will cause a change in the generation profile of the device. This leads to an imbalance in the individual sub-cell currents as the proportions of irradiance useful to each sub-cell are altered. In this work, the current generated by each cell is modeled simply by applying the spectral response of each sub-cell to the incident spectrum. This paper deals only with monolithic, two-terminal devices, hence the overall current is limited as equal to that of the sub-cell generating the least current, following Kirchhoff's current law.

The inputs required (in addition to those for the spectra! model) are the spectral response of each device to be modeled and measurements of short circuit current and irradiance spectra for validation. For the multi-junction devices, the spectral response data is required for each individual sub-cell, since the current contributions are modeled separately.

The devices modeled are single-junction and doublejunction sample modules from RWE Solar. Although the former are not intended for outdoor application, the arrangement allows the comparison of two different structures, but with a common manufacturing process.

The outdoor measurement system at CREST collects current-voltage curves from each PV test sample and irradiance spectra every ten minutes from sunrise to sunset, and meteorological data every minute. A PC running data acquisition software developed in-house handles logging and control. Where ambient air temperature, pressure and humidity data has not been available from the CREST system, measurements have been taken from the Loughborough University campus automated weather station, managed by the Geography department. Due to the slow temporal variation of these parameters, it is not thought that significant error is introduced through this approach.

For single-junction devices, spectral effects can be related to the UF. This can be viewed as a refinement of the resource available to a spectrally selective PV cell as the incident spectrum varies. This is a coarse approach which does not utilize the fine detail of the spectral response of the device under investigation, but has the advantage that it can be applied broadly to classes of device using the same type of material.

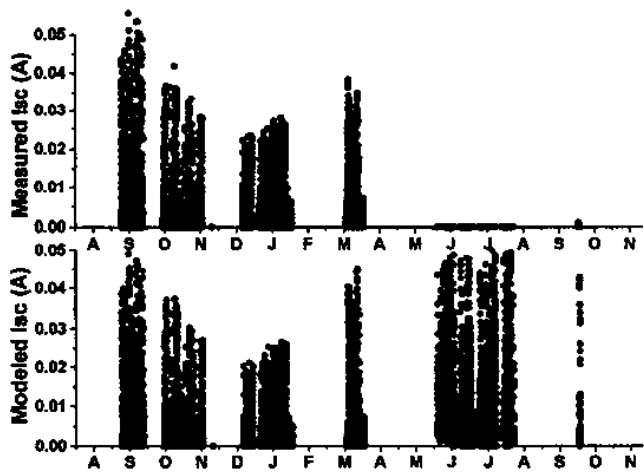

Fig. 3. Measured and modeled Isc over measurement period for the double-junction device

Multi-junction devices made of stacked cells of different band gaps exhibit a further (secondary) effect. The sub-cell current output must be necessarily matched for a single spectrum (typically, the AM1.5 standard), so when operating under illumination of differing spectra, the com- 
ponent cells become mismatched and the performance suffers.

\section{RESULTS}

Spectra and short circuit current were modeled only for those times where measurements existed to enable validation. The time period concerned is from August 2000 to November 2001. Measurements were not taken continuously because of the process of installing additional samples and instrumentation for other research projects and some unplanned outages. This can be seen in Fig. 3, which shows measured and modeled $l_{s c}$ for the double-junction device.

Fig. 4 shows the correlation between modeled and measured short circuit current for both the single- and double-junction device.
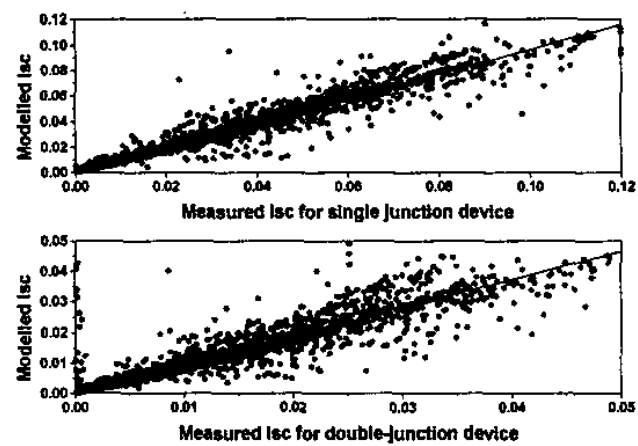

Fig. 4. Correlation of modeled and measured $\mathrm{l}_{\mathrm{sc}}$. Singlejunction top plot, double-junction bottom plot

The overall correlation is good with outliers mainly reflecting the relatively slow data collection rate when compared with changes in the operating conditions due to time variable weather. Applying a noise reduction aigorithm could certainly reduce these outliers. Even without this, the overall agreement is certainly significantly betterthan approaches that do not correct for spectral variation, Moreover, this approach is able to adequately model the non-linear behaviour of the short circuit current, as shown in [9].

\section{CONCLUSIONS}

It has been demonstrated that one can model the short circuit current of double-junction a-Si devices, provided spectral response data for each sub-cell is available and a reasonable picture of the incident spectrum is available. A model for the generation of incident spectral data is presented and applied to single and double junction devices. Coupling a relatively simple electrical model with the spectral input is shown to give good results. The common scatter when modeling of the $I_{S C}$ is significantly reduced. It is believed that this approach will allow to model the seasonal effects seen by single and double junction amorphous silicon devices.

\section{ACKNOWLEDGEMENTS}

The authors wish to thank Dr. H. Schade of RWE Solar, Division Phototronics, who supplied the samples and spectral response curves, and M. Sabry, for his valuable comments in discussion. Also many thanks to Steve Ransome for supporting Tom Betts' application for a travel grant from the Royal Academy of Engineering, and the Academy for making his attendance at the conference possible. This work is carried out with the financial support of the Engineering and Physical Sciences Research Council (EPSRC) of the United Kingdom through contract No. GR/N35694 and GR/N04232.

\section{REFERENCES}

[1] J. Merten, J. Andreu, "Clear Separation of Seasonal Effects on the Performance of Amorphous Silicon Solar Modules by Outdoor IN-Measurements", Solar Energy Materials and Solar Cells 52, 1998, pp. 11 25.

[2] J. A. d. Cueto, "Review of the Field Performance of One Cadmium Telluride Module", Progress in Photovoltaics: Research and Applications 6, 1998, pp. 433-446.

[3] T. Strand, et al, “Siemens Solar CIS Photovoltaic Module and System Performance at the National Renewable Energy Laboratory", Twenty-fifth IEEE PVSC, 1995, pp. 965-968.

[4] J. A. Eikelboom, M. J. Jansen, "Characterisation of PV Modules of New Generations: Results of Tests and Simulations", ECN-C-00-067, 2000.

[5] Y. Hirata, T. Tani, "Output Variation of Photovoltaic Modules with Environmental Factors - 1. The Effect of Spectral Solar Radiation on Photovoltaic Module Output", Solar Energy 55, 1995, pp.463-468.

[6] Y. Hirata et al, "Output Variation of Photovoltaic Modules with Environmental Factors - II. Seasonal Variation", Solar Energy 63, 1998, pp.185-189.

[7] R. Gottschalg et al, "Spectral Effects on the Performance of Thin Film Solar Cells at Loughborough, UK", UK-ISES Silver Jubilee Conference, 1999, pp. 35-41.

[8] R. E. Bird, C. Riordan, “Simple Solar Spectral Model for Direct and Diffuse irradiance on Horizontal and Tilted Planes at the Earth's Surface for Cloudless Atmospheres", Joumal of Climate and Applied Meteorology, 25, pp. 87-97.

[9] R. Gottschalg et a!, "Experimental Investigation of Spectral Effects On Amorphous Silicon Solar in Outdoor Operation", Twenty-Nineth IEEE PVSC, 2002, to be publ. 